\title{
O fim dos manicômios e a reinserção do paciente com transtorno mental na sociedade no âmbito do Distrito Federal, Brasil
}

The end of asylums and the reintegration of the patient with mental disorder in the society within the Federal District, Brazil

El fin de los manicomios y la reinserción del paciente con trastorno mental en la sociedad en el ámbito del Distrito Federal, Brasil

RESUMO: objetivo: identifica como a Reforma Psiquiátrica é aplicada no âmbito do Distrito Federal, analisando as instituições psiquiátricas mais tradicionais (Hospital São Vicente de Paulo e Instituto de Saúde Mental) que atuam no tratamento dos pacientes acometidos de transtornos mentais. Metodologia: trabalho descritivo-analítico, considerando que foi feita uma revisão teórico-crítica baseada em bibliografia jurídica, científica e médica, bem como em revisão bibliográfica de artigos já publicados sobre o tema. A ferramenta de análise foi o método analítico. Resultados: no Distrito Federal, ainda é insuficiente $o$ atendimento aos pacientes com transtornos mentais, pois a rede psiquiátrica é incipiente e inoperacional, não suprindo a demanda populacional que busca esses serviços como forma de tratamento. Conclusão: a saúde mental deve ser tratada de forma mais abrangente, englobando não somente os pacientes que apresentam sinais mais graves da doença, mas todos os que possam desenvolver algum transtorno mental.

Palavras-chave: Transtornos mentais. Serviços de Saúde Mental.

ABSTRACT: objective: this paper's purpose is to identify how the Psychiatric Reform is applied within the Federal District, analyzing the more traditional psychiatric institutions (St. Vincent de Paul Hospital and Institute of Mental Health) who work in the treatment of patients suffering from mental disorders. Methods: was been asked such patients pointing scientific studies showing that the patient has institutionalized mental health further aggravated and even then, public policy is not effective in patients reintegrate into society, though not the cure, but adapt to the social environment. Results: the work analyzed the structure of the Federal District to care for patients with mental disorders, as well as ways to verify the inclusion of these patients in society. Conclusion: there are critics vanishingly forms of psychiatric treatment of these users under those public institutions.

Keywords: Mental disorders. Mental Health Services

RESUMEN: objetivo: este trabajo ha buscado las instituciones psiquiátricas más tradicionales (Hospital São Vicente de Paulo e Instituto de Salud Mental) que actúan en el tratamiento de los pacientes acometidos de trastornos mentales. Metodología: se ha cuestionado el tratamiento de estos pacientes, apuntando estudios científicos que

\footnotetext{
${ }^{1}$ Advogado pelo Centro Universitário Estácio Brasília. E-mail: danilodireiton@yahoo.com.br
} 
demuestran que el paciente institucionalizado posee la salud mental aún más agravada y que, aún así, la política pública no es efectiva en reinsertar al paciente en la sociedad, aunque no lo cure, Pero lo adapte al medio social. Resultados: el trabajo presentó la estructura del Distrito Federal en la atención a los pacientes con trastornos mentales, así como verificar las formas de inserción de esos pacientes en la sociedad. Conclusión: hay críticas en cuanto a las formas de tratamiento de esos usuarios psiquiátricos en el ámbito de aquellas instituciones públicas.

Palabras Ilave: Trastornos mentales. Servicios de Salud Mental.

\section{Introdução}

A psiquiatria é um tema relevante para a sociedade, mas delicado, tendo em vista que a cultura predominante é que o paciente que possui transtorno mental, assim definido pela Lei 10.216 de 6 de abril de 2001 (1) seja institucionalizado e ali tratado, sem qualquer participação da sociedade e da família, com a visão voltada para a figura do médico e da medicalização do paciente, tornando os pacientes ociosos e sem uma perspectiva para a reinserção desses na sociedade.

Primeiramente, é abordado um contexto histórico da psiquiatria no Brasil e, de forma mais específica, no Distrito Federal, salientando fatos importantes que marcaram o tratamento na capital federal.

Logo após, discute-se sobre as leis federais que dão o embasamento legal para a Reforma Psiquiátrica, de suma importância devido ao caminho traçado para início da reforma.

Com essa discussão da legislação federal será realizado um breve resumo sobre a cobertura assistencial no Distrito Federal aos pacientes com transtornos mentais, com dados que demonstram a insuficiência ao atendimento, havendo, em seguida, críticas a esse sistema, destacando os problemas que persistem na atenção a esses pacientes.

Por fim, conclui-se o trabalho relacionando esses aspectos discutidos, com o modelo atual da política pública adotado pelo Governo do Distrito Federal e demonstrando outros aspectos relevantes para a melhora da atenção aos pacientes com transtornos mentais.

\section{Metodologia}

Tratou-se de um trabalho descritivo-analítico, considerando que foi feita uma revisão teórico-crítica acerca das discussões sobre as políticas públicas voltadas a reinserção de 
pacientes com transtornos mentais à sociedade, com a criação de Centros de Apoio Psicossocial, Residências Terapêuticas, atendimento-dia e internações, baseada em bibliografia jurídica, científica e médica, bem como em revisão bibliográfica de artigos já publicados sobre o tema. A ferramenta de análise foi o método analítico. Insta salientar que, no que toca aos procedimentos e técnicas de pesquisa, foi utilizada a pesquisa bibliográfica, legislativa e científica.

As variáveis consideradas no estudo foram a quantificação de instituições voltadas aos atendimentos psiquiátricos no Distrito Federal (demanda) e as formas de reinserção dos pacientes na sociedade por meio dessas instituições, que estão em número insuficiente, mas que fazem parte da proposta governamental voltada para saúde mental.

O mimeo oficial utilizado no trabalho foi fornecido pelo Ministério Público do Distrito Federal, que acompanha os aspectos da saúde mental no Distrito Federal.

\section{Questões históricas relevantes da Reforma Psiquiátrica no Distrito Federal}

$\mathrm{Na}$ década de 1960, quando Brasília foi inaugurada no governo de Juscelino Kubitschek, houve a unificação dos institutos de aposentadoria e pensões, sendo criado, o Instituto Nacional de Previdência Social (INPS). A partir daquele momento, o Estado iniciou a compra de serviços psiquiátricos do setor privado, tornando-se, portanto, a doença mental como objeto de lucro, o que aumentou consideravelmente o número de leitos e internações em hospitais psiquiátricos privados, chegando o Estado a destinar 97\% do total dos recursos da saúde mental somente para as internações hospitalares (2) .

Esse modelo contribuiu, significativamente, com a crise que assolou a Previdência Social no início dos anos 1980. A política pública ineficiente adotada pelo poder público, que não se preocupava em realizar um trabalho de transparência e decência ante a sociedade (2).

No Distrito Federal, o Hospital de Base foi a primeira instituição a criar uma Unidade Psiquiátrica que atendia, preferencialmente, pacientes com quadros psicóticos. Os considerados crônicos eram enviados a um sanatório localizado em Anápolis-GO (3). Essa situação, pois, "correspondeu a uma concepção higienista, de uma Brasília asséptica, onde não existiam loucos e nem lugar para eles" (4). 
Em 1987, surge um movimento que prega o fim dos manicômios, o Movimento dos Trabalhadores em Saúde Mental. Com o lema "Por uma sociedade sem Manicômios", mostrou a sociedade uma forma de discutir sobre loucura, doença mental, psiquiatria e seus manicômios. Sua finalidade é desinstitucionalizar e desospitalizar, desconstruindo a imagem de abandono da pessoa com transtorno mental (2).

Após esse debate e a promulgação da Constituição Federal de 1988, surge o Projeto de Lei 3657/89, que propõe, dentre outras medidas, o fim progressivo dos manicômios e sua substituição por outras modalidades assistenciais, o que se tornou realidade somente doze anos depois, com o advento da Lei no 10.216/2001 (1).

O marco da Reforma Psiquiátrica no Distrito Federal foi o fechamento da Clínica de Repouso Planalto - CRP, em 20 de março de 2001 (2), uma vez que foi a última clínica privada que mantinha um convênio com o SUS/DF, após denúncias de maus-tratos a pacientes, causando uma grande repercussão na mídia.

Com o fim da Clínica Planalto, em 2001, a reforma psiquiátrica no Distrito Federal tomou força e iniciou uma atenção mais sofisticada aos pacientes, possuindo como diretriz a Lei 10.216/01 (1), que fora sancionada 16 dias após esse episódio do fechamento da clínica.

\section{Breves comentários às Leis 10.216/01 e 10.708/03}

A Lei 10.216/01 dispõe sobre a proteção e os direitos das pessoas portadores de transtornos mentais e redireciona o modelo assistencial em saúde mental.

A lei trouxe como benefício o fim dos manicômios e da institucionalização dos pacientes, os quais apenas serão internados, em regime de exceção, quando os recursos extra-hospitalares se mostrarem insuficientes, como, por exemplo, a própria medicação não surtir efeito, trabalhos em grupo em que o paciente não interage, delegação de responsabilidade não recebida pelo paciente, dentre outras. Insta salientar, ainda, que a reinserção do paciente na sociedade é o foco principal dessa lei, tendo em vista que busca uma melhor qualidade de vida aos pacientes sob a sua ótica, dando à família e à sociedade um papel importante no tratamento desses pacientes. 
Nesse passo, a lei atendeu as exigências que há anos eram solicitadas pelos profissionais e pensadores estreitos com o tema, que sempre reivindicaram 0 fim dos manicômios por entenderem ser um tratamento desumano e que não atendia as necessidades dos pacientes.

A lei prevê, ainda, a criação de políticas públicas específicas para os pacientes que se encontram em situação de grave dependência institucional, com o fim de abarcar todos aqueles que estão em diferentes situações para a integração destes na sociedade.

O tratamento individual previsto reforça a ideia de que cada indivíduo possui uma necessidade e tratamento diferenciado, auxiliando-o em sua inserção na sociedade. Essa ideia coaduna com Machado (2), na qual afirma:

Tais dispositivos podem assegurar o rompimento da lógica manicomial, na medida em que suas práticas se voltem, de fato, para a construção de espaços de exercício de subjetividade singularizada, de respeito ao diferente e de busca pela inclusão das singularidades no âmbito social.

Havia ainda, a questão financeira, intimamente ligada à própria institucionalização do paciente, pois grande parte das famílias não possuem recursos suficientes para atender as necessidades do enfermo.

Para tanto, a Lei 10.708/03 (instituiu o auxílio-reabilitação psicossocial para pacientes acometidos de transtornos mentais egressos de internações, com o fim de reintegrar à sociedade o paciente, contribuindo com um auxílio pecuniário aos pacientes que satisfaçam as exigências legais, quais sejam: que o paciente seja egresso de internação psiquiátrica cuja duração tenha sido, comprovadamente, por um período igual ou superior a dois anos; que a situação clínica e social do paciente não justifique a permanência em ambiente hospitalar e indique tecnicamente a possibilidade de inclusão em programa de reintegração social e a necessidade de auxílio financeiro; expresso consentimento do paciente, ou de seu representante legal, em se submeter às regras do programa; que seja garantida ao beneficiado a atenção continuada em saúde mental, na rede de saúde local ou regional. 


\section{Instituições psiquiátricas e a reinserção do paciente na sociedade}

A expressão designada na Lei 10.216/01 (5) denomina "pessoas com transtornos mentais" aquelas pessoas que possuem algum déficit mental.

Todavia, essa expressão ainda causa certo preconceito, tendo em vista que essa nomenclatura não visa a inclusão do paciente na sociedade, mas apenas uma denominação, uma classe de pessoas que possuem problemas mentais que devem se submeter a um tratamento psiquiátrico. Essa expressão deveria causar um impacto mais positivo na sociedade, já como um meio de inserir e interagir com esses pacientes no meio social.

Nesse passo, é de se salientar que o transtorno mental, em seu contexto histórico, foi tratado como doença que deveria ser extirpada pela sociedade, na qual a internação dessas pessoas, por vezes compulsória, foi muito mais comum durante a idade média, pois segregava totalmente o doente da sociedade sem qualquer plano de tratamento e reinserção na sociedade.

Espiridião (5) aduz que a Reforma Psiquiátrica, em sua essência, visa criar ações para garantir a dignidade do paciente com transtorno mental:

Os movimentos precursores da Reforma Psiquiátrica brasileira, surgidos nos Estados Unidos e Europa a partir de meados do século XX, apontavam críticas ao atendimento dispensado aos portadores de doença mental, quando eram excluídos e segregados da sociedade, demandando ações com vistas a um atendimento mais humanizado, de forma a garantir sua dignidade, enquanto cidadão.

Nas últimas décadas, as políticas públicas voltadas à saúde mental têm procurado redirecionar seus parâmetros institucionais, reduzindo o número de leitos psiquiátricos em todo o Brasil e que logrou um avanço considerável com o advento das Lei 10.216/01 e 10.708/03, pois aumentou a rede de serviços de atendimento-dia (2).

Há certo consenso que a Lei 10.216/01 não supriu totalmente as necessidades dos pacientes com transtornos mentais, bem como o Poder Público não atingiu o objetivo que a lei propunha, qual seja, acabar com o modelo hospitalocêntrico, pois ainda se mantém o tratamento hospitalar como regra, e não exceção.

A Reforma Psiquiátrica possui o condão de reinserir as pessoas com transtornos mentais na sociedade (6): 


\begin{abstract}
A internação, portanto, é uma criação institucional própria ao século XVII e assume um sentido inteiramente diferente da prisão na Idade Média. É, assim, de uma invenção e não de uma evolução que Foucault trata. De um evento decisivo que rompe e modifica o sentido anteriormente reservado ao internamento. Um evento importante para a própria loucura, que agora é percebida no horizonte social da pobreza, da incapacidade para o trabalho e da impossibilidade de integrar-se ao grupo, modificando o seu sentido drasticamente. Nasce, assim, uma nova sensibilidade em relação à loucura, na qual esta é arrancada de sua liberdade imaginária tão presente na Renascença e se vê reclusa pelo internamento e ligada à Razão e às regras da moral.
\end{abstract}

O princípio geral da desinstitucionalização não é apenas retirar o paciente do hospital em que ficou internado, mas reinseri-lo na sociedade e fazer com que este exercite seu direito pleno de liberdade, cidadania, respeito à sua dignidade como pessoa, bem como os demais direitos elencados na Constituição Federal de 1988.

\title{
Cobertura assistencial de saúde mental no Distrito Federal
}

O Distrito Federal, por apresentar o pior indicador da Cobertura Assistencial CAPS do Brasil, por intermédio do seu governo, formulou uma Proposta de Ação para a Rede de Cuidados em Saúde Mental, Álcool e Outras Drogas no Âmbito da Secretaria de Saúde do Distrito Federal, com o fim de melhorar o atendimento e atenção aos portadores de transtornos mentais, bem como prover o estabelecido pela legislação federal e demais leis que tratam deste tema (7).

Essa proposta apresenta que entre $20 \%$ e $25 \%$ da população do Distrito Federal tiveram, têm ou terão algum tipo de transtorno mental, sendo que a sua população atual se encontra em torno de dois milhões e seiscentas mil pessoas, ou seja, seiscentas mil pessoas terão, alguma vez na vida, algum tipo de transtorno mental (6). Nesse contexto, é de salientar que a atenção à psiquiatria social voltada a essas pessoas é de extrema importância para a saúde da população, uma vez que estes transtornos podem afetar o seu convívio ante os demais indivíduos que o cercam.

Nesse passo, existem no Distrito Federal treze unidades CAPS ( Centro de Atendimento Psicossocial), assim distribuídas nas cidades de Taguatinga, Paranoá, Guará, Sobradinho, Ceilândia, Planaltina, Samambaia, Gama, Santa Maria, Rodoviária (em Brasília são dois CAPS), Centro de Orientação Médico Psicopedagógico e Riacho Fundo 
com o fim de dar esse suporte de atenção e acompanhamento ao paciente, contudo, insuficientes para a demanda existente.

Não há no Distrito Federal Residência Terapêutica, o que revela a falta de atenção especializada aos pacientes com transtornos mentais que, aliada à baixa cobertura dos CAPS, comprometem ainda mais esses atendimentos, conforme os próprios dados disponibilizados pela proposta de ação governamental (6).

Vale destacar ainda que há no DF 72 leitos para os pacientes com transtornos mentais oferecidos pelo Hospital São Vicente de Paulo e mais 24 leitos também para pacientes com transtornos mentais oferecidos pelo Hospital de Base, sendo que este último apenas atende aqueles pacientes que apresentam co-morbidades clínico-cirúrgicas. Destaca-se ainda que o Instituto de Saúde Mental abriga 30 pacientes, dentre eles detentos que cumprem medida de segurança e portadores de transtornos mentais severos (6).

A Portaria n. 336/GM/MS (8), de 19 de fevereiro de 2002, que trata dos CAPS e a quantidade de profissionais que estão em cada instituição, vislumbra-se que, nos termos da própria portaria, no CAPS II, instituição em maior número no DF, será atendido, no máximo, 45 pacientes ao dia, com uma equipe médica de doze profissionais, assim distribuídos: um médico, um enfermeiro com formação em saúde mental, quatro profissionais de nível superior compreendidos entre psicólogo, assistente social, enfermeiro, terapeuta ocupacional, pedagogo ou outro profissional necessário ao projeto terapêutico e seis profissionais de nível médio, compreendendo técnico e/ou auxiliar de enfermagem, técnico administrativo, técnico educacional e artesão.

Facilmente percebe-se a falta de estrutura para o atendimento integral e universal, pois estão defasados e insuficientes para atender à demanda requerida pela população no momento atual, pois somente em 2015 está prevista a implantação total de novas instituições psiquiátricas no DF.

\section{Críticas à cobertura assistencial de saúde mental no Distrito Federal}

As políticas públicas devem ser analisadas e aplicadas de forma efetiva, mas com cautela, tendo em vista que os transtornos mentais são uma questão delicada sob a ótica da sociedade, que segrega em razão de seu desconhecimento. Vale destacar, ainda, que 
o preconceito também faz parte da cultura da sociedade em extirpar o problema, que são os pacientes, com o fim de resolver a situação daquele meio, e não do doente.

Para tanto, a forma como são planejadas, implementadas, executadas e avaliadas essas políticas públicas são importantes no que toca à integração das instituições voltadas à saúde mental, tendo em vista que essa integração colabora com a ressocialização do paciente na sociedade.

O atendimento qualificado, tanto dos serviços como dos processos de trabalhos realizados no âmbito dessas instituições, colabora com a evolução dos tratamentos desses pacientes e em sua reinserção na sociedade, nos quais se deve privilegiar a produtividade baseada na qualidade, promovendo a inclusão social, econômica e cultural.

Nesse passo, os profissionais envolvidos na recuperação desses pacientes, bem como os próprios usuários, familiares, gestores, políticos, dentre outros, devem unir esforços para melhorar as condições em que os pacientes estão sujeitos, e banir a ideia da figura central do médico, medicação e manicômio como único meio de tratar um paciente com transtornos mentais.

Foucault (9) afirma que há inúmeras formas de lutas de resistência ao poder, destacando-se contra o assujeitamento, as de contestação e insubmissão. De acordo, pois, com esse pensamento, os pacientes não devem ser sujeitados à prisão manicomial, nem serem tratados de forma segregada, mas buscar um tratamento especializado que o adapte à sociedade.

Portanto, a discussão ampla das necessidades básicas, dos direitos e dos deveres da cidadania, deve acalentar os rumos que serão traçados com a Reforma Psiquiátrica, tendo em vista que os pacientes com transtornos mentais eram e são tratados como coisas, o que desrespeita a Constituição Federal de 1988.

Ainda, a fundamentação histórica, as concepções filosóficas e os contextos sociopolíticos e culturais são de suma importância para o tratamento desses pacientes, pois a experiência do passado colabora com o tratamento atual, afastando os erros e aplicando os acertos. A apropriação desses conhecimentos permite, pois, uma análise profunda, mais apropriada e eficaz para o avanço de um sistema de saúde onde a integralidade seja efetivamente aplicada, com uma produção social da saúde mais viável e com participação da sociedade, dos profissionais da saúde psiquiátrica e da família para sua construção. 
A Reforma Psiquiátrica privilegia, pois, a inclusão, a solidariedade e a cidadania. No Distrito Federal, a implantação dessa política se encontra em fase inicial, com muitas propostas e pouca efetividade, pois serão implantados, até 2015, cerca de quarenta e seis CAPS, conforme já salientado anteriormente, o que dificulta essa proposta da Reforma Psiquiátrica a ser aplicada de forma imediata, não olvidando que a lei diretriz que reformou a psiquiatria no Brasil é de meados de 2001, ou seja, há mais de dez anos foi criada e ainda não foi implantada.

Na proposta apresentada pelo GDF percebe-se que a rede de psiquiatria do governo está seriamente prejudicada, uma vez que em inúmeros momentos a Secretaria de Saúde do DF cede os médicos psiquiátricos a outros órgãos, bem como tem de disponibilizar quarenta horas semanais ao judiciário para atendimento de perícias cíveis e trabalhistas (6).

Vale destacar que Costa (7) ainda aponta que a atual situação está em nível crítico e restará inviável se não houver contratação urgente de profissionais de saúde para suprir essa deficiência, o que realça a falta de estrutura e atenção aos pacientes desse sistema, o que prejudica, também, a articulação entre as instituições, haja vista que a falta de pessoal torna o sistema deficiente.

O Hospital São Vicente de Paulo, localizado em Taguatinga - DF e referência no Distrito Federal no âmbito da psiquiatria, não possui uma articulação com as demais instituições psiquiátricas do DF, o que dificulta o tratamento dos pacientes. Tal afirmativa se consubstancia (10).

Outro resultado significativo, quanto à organização e funcionamento dos serviços internos do HSVP, CAPS e Hospital-dia, internação e emergência, é o fato de que estes não estão articulados entre si e com os demais serviços, mostrando-se insuficientes para atender às demandas dos usuários, com concentração de suas atividades na relação com o usuário e familiar. A organização das práticas de saúde mental para os serviços Hospital-dia e CAPS não se caracteriza como substitutivo de superação e transformação do modelo clínico-biológico, uma vez que não tem conseguido prescindir do hospital psiquiátrico.

Como bem demonstrado não há uma articulação com as instituições do Distrito Federal, o que dificulta no tratamento e inserção do paciente com transtorno mental na sociedade.

Lima e Silva (10) afirmam, sobre o Hospital São Vicente de Paulo: 
Entretanto, mesmo com as tentativas internas de expansão dos serviços, não se verifica caracterização dos princípios da reforma psiquiátrica, quando se analisa o conjunto de ações institucionais sob o enfoque do paradigma do modo psicossocial. Dessa forma, a experiência se mostra isolada, quando não articulada e integrada com a rede comunitária em saúde mental como uma política pública.

Percebe-se, pois, que a aplicação dos princípios da Reforma Psiquiátrica ainda é pouco efetiva neste hospital, com uma visão totalmente hospitalocêntrica.

Outra instituição de saúde mental que merece ser citada é o Instituto de Saúde Mental - ISM, localizado na região do Riacho Fundo. Essa instituição abriga 26 pacientes, provenientes da clínica com a qual a Secretaria de Estado de Saúde do DF mantinha um convênio, a Clínica de Repouso Planalto - CRP, que, após denúncias de maus-tratos, e com a intervenção do Ministério Público, foi fechada, sendo os vinte e seis pacientes transferidos para aquela instituição (3).

Ela ressalta, por conseguinte, que a maioria desses pacientes transferidos "não possuía mais qualquer vínculo familiar ou social identificável. Devido ao seu grau de comprometimento e cronificação, muitos já não tinham lembrança nem do próprio nome", bem como os registros se encontravam precários.

Ainda conforme Machado (3), foi instalada uma enfermaria provisória, onde seriam acolhidos os pacientes em, no máximo, três meses até que fossem montados os serviços residenciais terapêuticos do DF, fato este que até então não se concretizou e os pacientes se encontram até hoje no ISM.

Machado (3) afirma que:

A SES/DF está comprometida a criar os Serviços Residenciais Terapêuticos para acolher os usuários hoje abrigados no ISM e outros que também necessitam de tal dispositivo terapêutico. Tão logo os SRT sejam criados, a "Residência Terapêutica" do ISM, enquanto unidade provisória, deverá ser desativada.

Nesse contexto, entende-se que o Instituto de Saúde Mental não se enquadra no contexto de Residência Terapêutica, com caráter totalmente provisório e, conforme a proposta do Governo do Distrito Federal.

Nesse passo, com a política do DF voltada para o fim dos manicômios e, consequentemente, da contratação de leitos psiquiátricos privados conforme as diretrizes 
de saúde e as legislações federais vigentes, cabe ao governo implantar instituições terapêuticas com a finalidade de tratar os pacientes crônicos, há anos institucionalizados e dependentes do sistema.

Portanto, Machado (3) salienta que a falta de preparo dos funcionários atuantes no âmbito da saúde mental, bem como esses mesmos profissionais, por não acreditarem na inclusão social do paciente, tornam-se empecilhos graves que contribuem para a segregação desses pacientes com transtornos mentais.

Para tanto, a criação de instituições voltadas para a saúde mental, como o CAPS, RTs, dentre outras, são de fundamental importância para o tratamento e inclusão social do paciente, uma vez que essa criação tende a ser um desafio, na qual apenas a articulação entre a sociedade, o Estado e os profissionais, e porque não o próprio paciente, poderá contribuir para a recuperação e/ou inclusão desse paciente na sociedade.

Não podemos olvidar que, conforme Machado (3), a manutenção dos pacientes a longo prazo institucionalizados agrava a sua situação em relação ao transtorno mental, senão vejamos:

A institucionalização é justamente este percurso feito pelo interno dentro dos manicômios mediante o qual ele vai se tornando cada vez mais dependente da estrutura institucional como garantia de sua sobrevivência, em decorrência do isolamento social e da perda progressiva das capacidades e habilidades dos internos submetidos a rotinas disciplinadoras e despersonalizantes.

Portanto, a dependência do paciente ao sistema prejudica a sua recuperação, tornando-o vinculado à instituição, sem qualquer perspectiva de retorno à sociedade.

Nesse passo, a atenção integral à saúde, mais especificamente a mental, é contrária ao modo de atenção que se concentra no hospital psiquiátrico, que está intimamente ligado em sua trajetória de institucionalização para curar uma doença diagnosticada, o que demonstra a insuficiência para atender e tratar a complexidade da doença mental.

Nesse contexto, Machado (3) salienta:

O paciente institucionalizado pode, aos poucos, perder suas capacidades e habilidades, cronificando-o muito mais por ação do contexto hospitalar do que propriamente em função de sua patologia. É por esta razão que a ideia da reabilitação psicossocial nasce junto com a ideia de desinstitucionalização, ambas vão representar a busca pelo resgate da autonomia dos sujeitos em sofrimento mental, sua reinserção no contexto da família e da comunidade, no mundo da cultura, do trabalho e do lazer. 
A reabilitação psicossocial, como bem salientado pela autora, demonstra que a doença em si nem sempre é a causadora da incapacidade e vulnerabilidade do paciente, que, logo que é descoberta, é tratada com internação e sem qualquer participação efetiva da família, porém, essa visão tem mudado com a Reforma Psiquiátrica, pois a Lei 10.216/01 (1) põe fim aos manicômios e internações, exceto em alguns casos previstos na própria lei.

Contudo, o transtorno mental uma vez diagnosticado não necessariamente é tratado com um simples medicamento, mas toda uma gama de atividades para a recuperação do paciente, que poderá ser curado dependendo de sua situação, ou não, quando será avaliado e adaptado à sociedade, conforme afirma Machado (3).

No Distrito Federal, portanto, ainda é insuficiente o atendimento aos pacientes com transtornos mentais, uma vez que a rede psiquiátrica é incipiente e inoperacional, não suprindo a demanda populacional que busca esses serviços como forma de tratamento.

\section{Conclusão}

Conclui-se, após toda a exposição do trabalho, que a saúde mental deve ser tratada de forma mais abrangente, englobando não somente os pacientes que apresentam sinais mais graves da doença, mas todos os que, em decorrência de algum fato, desenvolve algum transtorno mental.

Cabe às políticas públicas, geridas pelo Estado, oferecer tratamentos específicos aos pacientes transtornados, principalmente àqueles que se encontram em situação de grave dependência institucional, abarcando, pois, todos aqueles que estão em diferentes situações para a reintegração e adaptação destes na sociedade.

O tratamento individual, com previsão legal nas legislações federais, reforça a ideia de que cada indivíduo, com sua particularidade, possui uma necessidade e tratamento diferenciado, o que auxilia sua inserção na sociedade. Nesse contexto, a Reforma Psiquiátrica privilegia, pois, a inclusão, a solidariedade e a cidadania desses pacientes que necessitam de um apoio não somente profissional, mas da sociedade e da família como um todo.

Uma dessas ações terapêuticas voltadas à integração do paciente na sociedade é a realização de oficinas de caráter profissionalizante, com a finalidade de capacitar 0 
paciente a torná-lo apto à vida laborativa, afastando, por completo, a incapacidade e a dependência da instituição internativa, oportunizando uma qualidade de vida superior a da institucionalização.

Os profissionais envolvidos na recuperação desses pacientes também possuem papel importante na adaptação destes na sociedade, pois devem abolir o referencial da medicação e manicômio como único meio de tratar um paciente com transtornos mentais, e aplicar outros meios eficazes, como as ações terapêuticas.

Essas ações terapêuticas, em conjunto com a sociedade e a família, proporcionam aos pacientes uma qualidade de vida melhor, uma vez que não se busca a cura, o que em alguns casos não é possível, mas uma adaptação ao meio em que o paciente está inserido.

Nesse passo, o Distrito Federal e a sua política atual que visa o fim dos manicômios, baseando-se nas legislações federais, deve implantar alternativas institucionais com o fim de tratar as pessoas acometidas com transtornos mentais, uma vez que, conforme dados do Ministério Público citados neste trabalho, de vinte a vinte e cinco por cento da população teve, tem ou terá algum transtorno psíquico.

O fim dos manicômios, como forma de tratamento dos pacientes acometidos de transtornos mentais, não é apenas desinstitucionalizá-lo do hospital em que ficou internado, mas reinseri-lo na sociedade e fazer com que este exercite seu direito pleno de liberdade, cidadania e respeito à sua dignidade como pessoa, e não por sua coisificação.

Vale destacar que não existe no âmbito do Distrito Federal, conforme salientado anteriormente, Residência Terapêutica, instituição esta a responsável para a inclusão social do paciente na sociedade, tratando-o conforme sua necessidade e individualidade. Não se deve olvidar que essa falta de atenção especializada, concomitante à baixa cobertura de outras instituições psiquiátricas no Distrito Federal, prejudica enormemente a recuperação, adaptação e reinserção desses pacientes na sociedade.

Portanto, o Governo do Distrito Federal, por apresentar o pior índice de instituições psiquiátricas por habitantes do Brasil, requer uma atenção mais aprofundada na saúde mental, investindo não somente em instituições e profissionais da saúde, mas também em outros meios de fomentar a adaptação dos pacientes com transtornos mentais na sociedade. 
Portanto, em razão da não aplicação efetiva da Reforma Psiquiátrica no Distrito Federal proposta principalmente pela Lei no 10.216/01, percebe-se que os pacientes com transtornos mentais estão desassistidos, pois não há instituições suficientes que os acolham, bem como não há uma política voltada para a reinserção e adaptação desses pacientes na sociedade, prejudicando em sua qualidade de vida, pois eles tornam-se dependentes do sistema e não possuem qualquer expectativa em relação a própria vida.

Por fim, a simples criação de instituições psiquiátricas não soluciona o problema dos pacientes com transtornos mentais, mas sim haveria uma melhora significativa desses pacientes se existisse uma política pública voltada, primeiramente e antes de qualquer forma de remediação, à prevenção, o que é totalmente inexistente nessas políticas.

\section{Referências}

1.Brasil. Lei $n^{0} 10.216$, de 06 de abril de 2001. Dispõe sobre a proteção e os direitos das pessoas portadoras de transtornos mentais e redireciona o modelo assistencial em saúde mental. Disponível em <http://www. planalto.gov.br/ccivil_03/Leis/LEIS_2001/L10216.htm> Acesso em: 26/10/12.

2.Amarante, PDC. Psiquiatria Social e Reforma Psiquiátrica. Rio de Janeiro: FIOCRUZ, 1994.

3. Machado, DM A desconstrução do manicômio interno como determinante para a inclusão social da pessoa em sofrimento mental. Brasília, 2006.

4. Faleiros, E. 40 Anos de loucura no Distrito Federal: história breve dos serviços públicos de saúde mental no Distrito Federal. Brasília, 2000.

5.Esperidião, E. Assistência em saúde mental. A inserção da família na assistência psiquiátrica. Disponível: <http://www.fen.ufg.br/fen_revista/revista3_1/samental.html>. Acesso em 29/10/2012.

6.Vieira, PP. Reflexões sobre A História da Loucura de Michel Foucault. Disponível em: <http://www.unicamp.br/ aulas/pdf3/24.pdf> Acesso em: 30/10/2012.

7.Costa-Rosa, A. O modo psicossocial: um paradigma das práticas substitutivas ao modo asilar. In: Amarante, Paulo Duarte de Carvalho (org.). Ensaios: subjetividade, saúde mental, sociedade. Rio de Janeiro: Fiocruz, 2000, p. 314.8.

8.Brasil. Portaria/GM no 336, de 19 de fevereiro de 2002. Portaria que define e estabelece diretrizes para o funcionamento dos Centros de Atenção Psicossocial. Estes serviços passam a ser categorizados por porte e clientela, recebendo as denominações de CAPS I, 
Cuadernos Iberomericanos

de Derecho Sanitario

CAPS II, CAPS III, CAPSi e CAPSad. Disponível em

<http://www.inverso.org.br/index.php/content/view/4125.html> Acesso em: 04/11/12.

9. Foucault, M. Microfísica do Poder. Rio de Janeiro: Graal, 2008.

10. Lima, MG; Silva, GB. A Reforma Psiquiátrica no Distrito Federal. Disponível em: <http://www.scielo.br/scielo.php?script=sci_arttext\&pid=S0034-71672004000500015> Acesso em: 04/11/2012.

Submetido em: 30.1.2012

Re-submetido em:5.6.2017

Aprovado em: 29.6.2017

Pinto SD. O fim dos manincômios e a reinserção do paciente com transtorno mental na sociedade no âmbito do Distrito Federal, Brasil. Revista Cadernos Ibero-Americanos de Direito Sanitário. 2017 abr./jun, 6(2):95110. 